\title{
BETTI NUMBERS OF TORIC IDEALS OF GRAPHS: A CASE STUDY
}

\author{
FEDERICO GALETTO, JOHANNES HOFSCHEIER, GRAHAM KEIPER, CRAIG KOHNE, \\ MIGUEL EDUARDO URIBE PACZKA, AND ADAM VAN TUYL
}

\begin{abstract}
We compute the graded Betti numbers for the toric ideal of a family of graphs constructed by adjoining a cycle to a complete bipartite graph. The key observation is that this family admits an initial ideal which has linear quotients. As a corollary, we compute the Hilbert series and $h$-vector for all the toric ideals of graphs in this family.
\end{abstract}

\section{INTRODUCTION}

Let $G$ be any finite simple graph with edge set $E(G)=\left\{e_{1}, \ldots, e_{q}\right\}$ and vertex set $V(G)=\left\{x_{1}, \ldots, x_{n}\right\}$. We define the polynomial rings $k[V(G)]=k\left[x_{1}, \ldots, x_{n}\right]$ and $k[E(G)]=k\left[e_{1}, \ldots, e_{q}\right]$ for any field $k$, and the ring homomorphism

$$
\phi_{G}: k[E(G)] \rightarrow k[V(G)] \text { by } e_{i} \mapsto x_{j} x_{k} \text { if } e_{i}=\left\{x_{j}, x_{k}\right\} .
$$

The kernel of the map $\phi_{G}$, denoted by $I_{G}=\operatorname{ker}\left(\phi_{G}\right)$, is called the toric ideal associated with the graph $G$. It is well-known that $I_{G}$ is a homogeneous ideal generated by binomials (see [9, Prop. 5.19]), and in particular, the generators of $I_{G}$ correspond to closed even walks in $G$ (see [24], and [20, 23] for a detailed analysis of the minimal generators).

Associated with $I_{G} \subseteq R=k[E(G)]$ is a minimal graded free resolution of the form:

$$
0 \rightarrow \bigoplus_{j} R(-j)^{\beta_{l, j}\left(I_{G}\right)} \rightarrow \bigoplus_{j} R(-j)^{\beta_{l-1, j}\left(I_{G}\right)} \rightarrow \cdots \rightarrow \bigoplus_{j} R(-j)^{\beta_{0, j}\left(I_{G}\right)} \rightarrow I_{G} \rightarrow 0
$$

where $l \leq q$ and $R(-j)$ is the free $R$-module obtained by shifting the degrees of $R$ by $j$ (i.e., so that $\left.R(-j)_{a}=R_{a-j}\right)$. The number $\beta_{i, j}\left(I_{G}\right)$ is called the $i, j$-th graded Betti number of $I_{G}$ and equals the number of minimal generators of degree $j$ in the $i$-th syzygy module of $I_{G}$. Ideally, we would like to determine $\beta_{i, j}\left(I_{G}\right)$ directly from a description of $G$. A non-exhaustive list of papers that have worked towards a dictionary between the invariants of the minimal resolution and $G$ includes 1 , 2, 17, 8, 10, 13, 14, 16, 18, 20, 24. However, given that it can be difficult to enumerate the minimal generators of $I_{G}$, i.e., the numbers $\beta_{0, j}\left(I_{G}\right)$, a general solution to this problem is currently unknown.

Given the difficulty of finding $\beta_{i, j}\left(I_{G}\right)$ for arbitrary graphs, one is led to study restricted families. In this paper we carry out a case study of a special family of graphs, and we show that for this family, we can determine all of the graded Betti numbers of the corresponding toric ideal. In particular, we focus on a family of graphs, denoted $G_{r, d}$, that is constructed from the complete bipartite graph $K_{2, d}$ with $d \geq 2$, and joining the two vertices of degree

2000 Mathematics Subject Classification. 13D02, 13P10, 14M25, 05E40.

Key words and phrases. Toric ideals, graphs, graded Betti numbers, Hilbert series, Gröbner bases.

Version: November 7, 2018. 


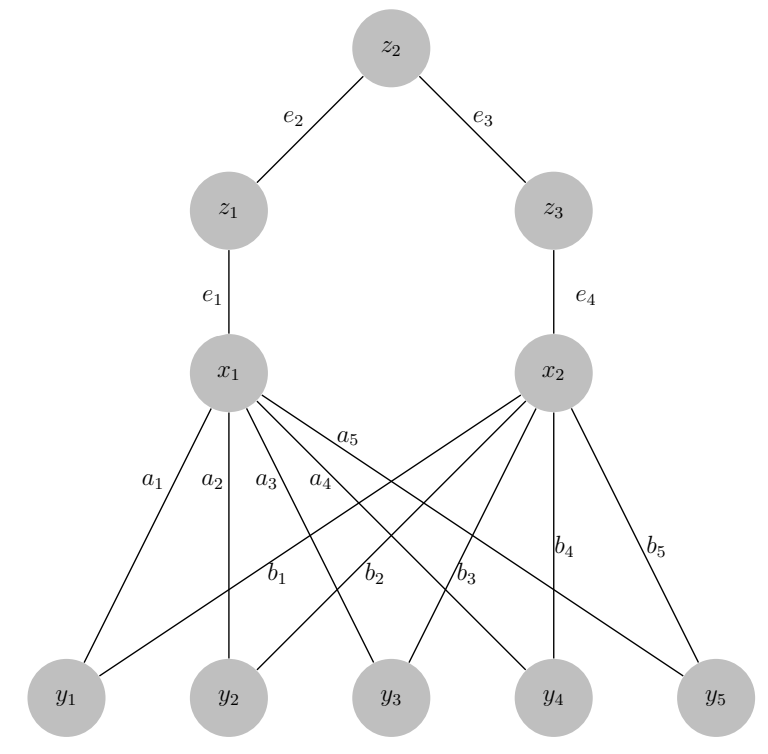

FIGURE 1. Illustration of $G_{3,5}$

$d$ with an even path of length $2 r-2$ with $r \geq 3$ (see Figure 1 for the graph $G_{3,5}$ ). These graphs are especially interesting with regard to Stanley's conjecture which asks whether any graded Cohen-Macaulay domain has a unimodal $h$-vector. We refer to Remark 4.4 for further details and references. From this point of view the graphs $G_{r, d}$ are appealing because the associated Cohen-Macaulay domains $k[E(G)] / I_{G}$ have $h$-vector $(1, d, \ldots, d)$ (see Theorem 4.3). The question now is whether one can modify the very tractable graphs $G_{r, d}$ in such a way that the associated Cohen-Macaulay domain is still normal, but its $h$-vector is no longer unimodal. The quest for such examples is an active area of research in Ehrhart theory and similar approaches have been already made (see [4]).

To compute the graded Betti numbers, we find a monomial order such that the corresponding initial ideal $J=\operatorname{in}\left(I_{G_{r, d}}\right)$ is a square-free monomial ideal. We exploit the fact that $\beta_{i, j}\left(I_{G_{r, d}}\right) \leq \beta_{i, j}(J)$, and that for all $G_{r, d}$, the ideal $J$ has linear quotients whose graded Betti numbers can be computed using [21].

The approach of using the initial ideal was also employed in [2]; we hope this case study further illustrates the usefulness of this technique to compute (or bound) graded Betti numbers of toric ideals, especially since we know of very few families of graphs where we know all the values $\beta_{i, j}\left(I_{G}\right)$. Our work is in the spirit of Conca and Varbaro's paper [6] which shows that many of the homological invariants of an ideal are related to its initial ideal if the initial ideal is square-free. Note that our special family can give us some partial insight into the graded Betti numbers of $I_{G}$ for more general graphs. In particular, if $G_{r, d}$ is an induced subgraph of $G$, we can apply work of Beyarslan, Hà, and O'Keefe [1] to show $\beta_{i, j}\left(I_{G_{r, d}}\right) \leq \beta_{i, j}\left(I_{G}\right)$; Theorem 4.1 uses this inequality to give lower bounds on the Castelnuovo-Mumford regularity and projective dimension for any toric ideal $I_{G}$.

We have organized the paper as follows. In Section 2, we recall the necessary preliminaries. In Section 3, we provide an explicit formula for the graded Betti numbers of the 
BETTI NUMBERS OF TORIC IDEALS OF GRAPHS: A CASE STUDY

toric ideal of the graphs $G_{r, d}$ (see Theorem 3.9). Finally, in Section 4, among other things, we compute the Hilbert series for the ring $k\left[E\left(G_{r, d}\right)\right] / I_{G_{r, d}}$ (see Theorem 4.3).

Acknowledgments. This paper started as a class project for a graduate reading course on toric ideals. Paczka thanks CONACYT for financial support and the hospitality of McMaster University. Van Tuyl's research was supported by NSERC Discovery Grant 2014-03898. Experiments with Macaulay2 [11] led to many of our results. We would also like to thank Jennifer Biermann, Tina O'Keefe, and the referee for their feedback.

\section{BACKGROUND}

We recall the relevant graph theory and commutative algebra background.

2.1. Simple graphs, walks, and binomials. Let $G$ be a graph with vertex set $V(G)=$ $\left\{x_{1}, \ldots, x_{n}\right\}$ and edge set $E(G)=\left\{e_{1}, \ldots, e_{q}\right\}$. For each edge $e_{i}$ there is an associated pair of distinct vertices $\left\{x_{i_{1}}, x_{i_{2}}\right\}$; we will use $e_{i}$ and $\left\{x_{i_{1}}, x_{i_{2}}\right\}$ interchangeably. All graphs are assumed to be finite simple graphs, i.e., $|V(G)|<\infty$ and there is at most one edge between any pair of vertices.

A walk $W$ of length $r$ is a sequence of edges $W=\left(\left\{v_{0}, v_{1}\right\},\left\{v_{1}, v_{2}\right\}, \ldots,\left\{v_{r-1}, v_{r}\right\}\right)$, where the $v_{j}$ are vertices in $V(G)$ and the $\left\{v_{j}, v_{j+1}\right\}$ are the edges connecting $v_{j}$ and $v_{j+1}$. A walk is a closed walk if $v_{0}=v_{r}$, and it is an even walk if $r$ is even. We say a closed walk $W=\left(e_{0}, e_{1}, \ldots, e_{r-1}\right)$ is a minimal closed walk if no two consecutive (modulo $r$ ) edges are equal.

The generators of the toric ideal of $I_{G}$ are binomials that can be described in terms of closed even walks in $G$. Specifically, if $W=\left(\left\{v_{0}, v_{1}\right\},\left\{v_{1}, v_{2}\right\}, \ldots,\left\{v_{2 s-1}, v_{2 s}=v_{0}\right\}\right)$ is a closed even walk, we associate with $W$ the binomial

$$
f_{W}=\prod_{j=1}^{s} e_{2 j-1}-\prod_{j=1}^{s} e_{2 j} \in k\left[e_{1}, \ldots, e_{q}\right]
$$

where $e_{j}=\left\{v_{j-1}, v_{j}\right\}$ for $1 \leq j \leq 2 s$. A binomial $f=u-v \in I_{G}$ is called a primitive binomial if there is no other binomial $g=u^{\prime}-v^{\prime} \in I_{G}$ such that $u^{\prime} \mid u$ and $v^{\prime} \mid v$. A closed even walk $W$ of $G$ is a primitive walk if its associated binomial $f_{W}$ is a primitive binomial. The following observation will be useful in the sequel.

Theorem 2.1. [9, Proposition 5.19] Let $G$ be a finite simple graph. Then the set of primitive walks in $G$ is a Gröbner basis of $I_{G}$ with respect to any monomial ordering.

The next lemma follows directly from the definitions.

Lemma 2.2. Let $G$ be a finite simple graph.

(i) All primitive walks of $G$ are minimal walks.

(ii) A closed even walk $W$ of $G$ which can be expressed as two consecutive closed even walks, i.e.,

$W=\left(\left\{x_{1,1}, x_{1,2}\right\},\left\{x_{1,2}, x_{1,3}\right\}, \ldots,\left\{x_{1, n}, x_{1,1}\right\},\left\{x_{2,1}, x_{2,2}\right\},\left\{x_{2,2}, x_{2,3}\right\}, \ldots,\left\{x_{2, m}, x_{2,1}\right\}\right)$ with $x_{1,1}=x_{2,1}$, is not primitive. 
2.2. Linear quotients, initial ideals, and Hilbert series. We recall some general results about homogeneous ideals $I$ in a polynomial ring $S=k\left[z_{1}, \ldots, z_{n}\right]$. Ideals with linear quotients, which play a key role in our main theorem, were introduced by Herzog and Takayama [12]; a more general definition can be found in Sharifan and Varbaro [21].

Definition 2.3. Let $I \subseteq S$ be a homogeneous ideal with an ordered minimal set of homogeneous generators $\left\{f_{1}, \ldots, f_{m}\right\}$. Then $I$ has linear quotients with respect to $\left\{f_{1}, \ldots, f_{m}\right\}$ if each ideal quotient $\left\langle f_{1}, \ldots, f_{j-1}\right\rangle:\left\langle f_{j}\right\rangle$ for $j=2, \ldots, m$ is generated by linear forms.

When $I$ has linear quotients, the graded Betti numbers are a function of the number of generators of the ideal quotients.

Theorem 2.4 ([21, Corollary 2.7]). Let $I \subseteq S$ be a homogeneous ideal with linear quotients with respect to $f_{1}, \ldots, f_{m}$ where $\left\{f_{1}, \ldots, f_{m}\right\}$ is a minimal system of homogeneous generators for $I$. Let $n_{p}$ be the minimal number of homogeneous generators of $\left\langle f_{1}, \ldots, f_{p-1}\right\rangle:\left\langle f_{p}\right\rangle$ for $p=1, \ldots, m$. Then

$$
\beta_{i, i+j}(I)=\sum_{1 \leq p \leq m, \operatorname{deg}\left(f_{p}\right)=j}\left(\begin{array}{c}
n_{p} \\
i
\end{array}\right)
$$

Fix a monomial order $<$ on the ring $S$. If $I$ is any homogeneous ideal, then the initial ideal of $I$, denoted $\operatorname{in}_{<}(I)$ (or in $(I)$ if $<$ is clear), is the monomial ideal generated by the initial terms of the elements of $I$. We shall need the following result:

Theorem 2.5 ([19, Theorem 22.9]). Fix any monomial order $<$ on $S$. If $I \subseteq S$ is a homogeneous ideal, then $\beta_{i, j}(I) \leq \beta_{i, j}\left(\operatorname{in}_{<}(I)\right)$ for all $i, j \geq 0$.

For a homogeneous ideal $I \subseteq S$, the Hilbert series of $S / I$ is the generating function

$$
H S_{S / I}(t)=\sum_{i=0}^{\infty} \operatorname{dim}_{k}(S / I)_{i} t^{i}=\frac{Q(t)}{(1-t)^{d}}
$$

where $Q(t) \in \mathbb{Z}[t], d=\operatorname{dim} S / I$, and $\operatorname{dim}_{k}(S / I)_{i}$ is the dimension of the $i$-th graded piece of $S / I$. If $Q(t)=h_{0}+h_{1} t+\cdots+h_{r} t^{r}$, then the $h$-vector is of $S / I$ is $h=\left(h_{0}, \ldots, h_{r}\right)$. The next lemma allows to deduce when $I$ and in $_{<}(I)$ have the same graded Betti numbers.

Lemma 2.6. Fix any monomial order $<$ on $S$. Let $I \subseteq S$ be a homogeneous ideal, and let $J=\operatorname{in}_{<}(I)$. Suppose that there exists an integer $k$ such that $\beta_{i, i+j}(I)=\beta_{i, i+j}(J)$ for all $i$ and all $j \neq k$. Then we also have $\beta_{i, i+k}(I)=\beta_{i, i+k}(J)$ for all $i \geq 0$.

Proof. Let $b_{i, i+j}=\beta_{i, i+j}(I)$ and $c_{i, i+j}=\beta_{i, i+j}(J)$. The ideals $I$ and $J$ have the same Hilbert series (see [9, Proposition 4.29]), which can be expressed in terms of their graded Betti numbers via the formulas (see [9, Proposition 4.27]):

$$
H S_{S / I}(t)=\frac{\sum_{i}(-1)^{i}\left(\sum_{j} b_{i, i+j} t^{i+j}\right)}{(1-t)^{n}}, \text { and } H S_{S / J}(t)=\frac{\sum_{i}(-1)^{i}\left(\sum_{j} c_{i, i+j} t^{i+j}\right)}{(1-t)^{n}}
$$


Our hypotheses imply that $b_{i, i+j}=c_{i, i+j}$ for all $i$ and $j$ if $j \neq k$. Since $H S_{R / I}(t)=$ $H S_{R / J}(t)$, after this substitution and simplifying, we arrive at

$$
\sum_{i}(-1)^{i} b_{i, i+k} t^{i+k}=\sum_{i}(-1)^{i} c_{i, i+k} t^{i+k}
$$

Comparing the coefficients on both sides gives the desired identity.

\section{The FAMILY of GRAPHS $G_{r, d}$ AND THEIR TORIC IDEALS}

We now define the family of graphs $G_{r, d}$ which will be the focus of our case study. Given positive integers $r \geq 3$ and $d \geq 2$, the graph $G_{r, d}$ has vertex set

$$
V\left(G_{r, d}\right)=\left\{x_{1}, x_{2}, y_{1}, \ldots, y_{d}, z_{1}, \ldots, z_{2 r-3}\right\}
$$

and edge set

$$
\begin{aligned}
E\left(G_{r, d}\right)=\{ & \left.\left\{x_{i}, y_{j}\right\} \mid 1 \leq i \leq 2,1 \leq j \leq d\right\} \cup\left\{\left\{x_{1}, z_{1}\right\},\left\{x_{2}, z_{2 r-3}\right\}\right\} \\
& \cup\left\{\left\{z_{i}, z_{i+1}\right\} \mid 1 \leq i \leq 2 r-4\right\} .
\end{aligned}
$$

We label the edges of $G_{r, d}$ as follows. Let $e_{1}=\left\{x_{1}, z_{1}\right\}, e_{2 r-2}=\left\{z_{2 r-3}, x_{2}\right\}$ and for $i \in\{2, \ldots, 2 r-3\}$ let $e_{i}=\left\{z_{i-1}, z_{i}\right\}$. For $i \in\{1, \ldots, d\}$ let $a_{i}=\left\{x_{1}, y_{i}\right\}$ and $b_{i}=$ $\left\{x_{2}, y_{i}\right\}$. Informally, the graph $G_{r, d}$ is the complete bipartite graph $K_{2, d}$ (the graph with $V\left(K_{2, d}\right)=\left\{x_{1}, x_{2}, y_{1}, \ldots, y_{d}\right\}$ and $\left.\left.E\left(K_{2, d}\right)=\left\{\left\{x_{i}, y_{j}\right\} \mid 1 \leq i \leq 2,1 \leq j \leq d\right\}\right)\right)$ where we have added a path of length $2 r-2$ between the two vertices of degree $d$ in $K_{2, d}$. See Figure 1 in the introduction for the graph $G_{3,5}$ equipped with our labelling.

Remark 3.1. It is prudent to discuss the boundary cases. Note that if $r=1,2$ or if $d=1$, then we can still define $G_{r, d}$. If $r=1$, then we are adding a path of length zero to $K_{2, d}$, and thus $G_{1, d}=K_{2, d}$. If $r=2$, then $G_{2, d}=K_{2, d+1}$. Since the graded Betti numbers of $I_{K_{2, d}}$ are known (and we will give a new proof) we can assume throughout that $r \geq 3$. Furthermore, if $d=1$, then $G_{r, 1}$ is a cycle of length of $2 r$ if $r \geq 2$, or $G_{1,1}=K_{2,1}$. In the first case, $I_{G_{r, 1}}$ is a principal ideal whose Betti numbers are again known. In the second case, $I_{G_{1,1}}=\langle 0\rangle$. We can thus restrict our discussion to $r \geq 3$ and $d \geq 2$.

We can give an explicit description of the primitive walks of $G_{r, d}$. Note that we say two closed even walks $W=\left(e_{0}, \ldots, e_{2 s-1}\right)$ and $W^{\prime}=\left(e_{0}^{\prime}, \ldots, e_{2 s-1}^{\prime}\right)$ are equivalent up to a circular permutation if there exists an $i$ such that $e_{j}=e_{j+i}^{\prime}$ (or $e_{j}=e_{(2 s-j)+i}^{\prime}$ if the cycle is in the reverse order) for all $j$ where $j+i$ (or $(2 s-j)+i$ ) is taken modulo $2 s$.

Lemma 3.2. Fix integers $r \geq 3, d \geq 2$. Let $W$ be a primitive walk of $G_{r, d}$. Then, up to a circular permutation, $W$ is of one of the following:

(i) $W=\left(a_{i}, b_{i}, b_{j}, a_{j}\right)$ where $1 \leq i<j \leq d$, or

(ii) $W=\left(a_{i}, e_{1}, e_{2}, \ldots, e_{2 r-2}, b_{i}\right)$ where $1 \leq i \leq d$.

Proof. Observe that if we delete $x_{1}$ or $x_{2}$ from $G_{r, d}$, then we are left with a tree. Any closed even walk on a tree is not minimal and cannot be primitive by Lemma 2.2. This means $W$ must pass through $x_{1}$ and $x_{2}$. Further, $W$ must pass through $x_{1}$ and $x_{2}$ exactly once, otherwise $W$ can be expressed as two consecutive closed even walks, and so it is 
not primitive by Lemma 2.2. We can determine $W$ by selecting two edges adjacent to $x_{1}$. Since $W$ is minimal, the selected edges must be distinct. There are two cases.

In the first case, we select edges $\left\{a_{i}, a_{j}\right\}$ adjacent to $x_{1}$ where $i \neq j$. Then a primitive walk passing through $x_{1}$ and $x_{2}$ exactly once is of the form $\left(a_{i}, b_{i}, b_{j}, a_{j}\right)$. In the second case, we select edges $\left\{a_{i}, e_{1}\right\}$ adjacent to $x_{1}$. Then a primitive walk passing through $x_{1}$ and $x_{2}$ exactly once is of the form $\left(a_{i}, e_{1}, e_{2}, \ldots, e_{2 r-2}, b_{i}\right)$.

This describes the primitive walks of $G_{r, d}$ up to a circular permutation.

Corollary 3.3. Fix integers $r \geq 3$ and $d \geq 2$. A Gröbner basis for $I_{G_{r, d}}$ with respect to any monomial ordering is given by

$$
\left\{a_{i} b_{j}-b_{i} a_{j} \mid 1 \leq i<j \leq d\right\} \cup\left\{a_{i} e_{2} e_{4} \cdots e_{2 r-2}-b_{i} e_{1} e_{3} e_{5} \cdots e_{2 r-3} \mid 1 \leq i \leq d\right\} .
$$

Proof. This corollary follows from Theorem 2.1 and Lemma 3.2. Note that while Theorem 2.1 specifies all primitive walks, if $W$ and $W^{\prime}$ are two primitive walks that are equivalent up to circular permutation, then $f_{W} \in I_{G}$ if and only if $f_{W^{\prime}} \in I_{G}$.

Remark 3.4. Note that the set $\left\{\left(a_{i}, b_{i}, b_{j}, a_{j}\right) \mid 1 \leq i<j \leq d\right\}$ is the set of all primitive walks, up to circular permutation, of the complete bipartite graph $K_{2, d}$. By Theorem 2.1 the set $\left\{a_{i} b_{j}-b_{i} a_{j} \mid 1 \leq i<j \leq d\right\}$ is a Gröbner basis of the toric ideal $I_{K_{2, d}}$ with respect to any monomial order.

Let $>$ denote the graded reverse lex order on the polynomial ring $k\left[a_{1}, \ldots, a_{d}, b_{1}, \ldots, b_{d}\right.$, $\left.e_{1}, \ldots, e_{2 r-2}\right]$ where the variables are ordered in the following way:

$$
a_{1}>\cdots>a_{d}>e_{1}>\cdots>e_{2 r-2}>b_{1}>\cdots>b_{d} .
$$

Going forward, we let in $\left(I_{G_{r, d}}\right)$ denote the initial ideal of $I_{G_{r, d}}$ with respect to $>$. Let $\mathcal{G}_{r, d}$ denote the unique minimal generating set of $\operatorname{in}\left(I_{G_{r, d}}\right)$. It follows from Corollary 3.3 that $\mathcal{G}_{r, d}=F_{d} \cup H_{r, d}$ where

$$
F_{d}=\left\{a_{i} b_{j} \mid 1 \leq j<i \leq d\right\} \text { and } H_{r, d}=\left\{a_{i} e_{2} e_{4} \cdots e_{2 r-2} \mid 1 \leq i \leq d\right\} .
$$

Each monomial of $F_{d}$ has degree two and each monomial of $H_{r, d}$ has degree $r$. Also

$$
\left|\mathcal{G}_{r, d}\right|=\left|F_{d}\right|+\left|H_{r, d}\right|=\left(\begin{array}{l}
d \\
2
\end{array}\right)+d=\left(\begin{array}{c}
d+1 \\
2
\end{array}\right)=\frac{d(d+1)}{2} .
$$

Our immediate goal is to show that $\operatorname{in}\left(I_{G_{r, d}}\right)$ has linear quotients. We order the generators in $\mathcal{G}_{r, d}$ from least to greatest using the graded reverse lex order, and write these elements as $m_{1}, \ldots, m_{\frac{d(d+1)}{2}}$. That is,

$$
\begin{aligned}
F_{d} & =\left\{m_{1}, \ldots, m_{\frac{d(d-1)}{2}}\right\} \\
& =\{\underbrace{a_{d} b_{d-1}}_{b_{d-1} \text { as factor }}, \underbrace{a_{d} b_{d-2}, a_{d-1} b_{d-2}}_{b_{d-2} \text { as factor }} \underbrace{a_{d} b_{d-3}, a_{d-1} b_{d-3}, a_{d-2} b_{d-3}}_{b_{d-3} \text { as factor }}, \ldots, \underbrace{a_{d} b_{1}, a_{d-1} b_{1}, \ldots, a_{2} b_{1}}_{b_{1} \text { as factor }}\},
\end{aligned}
$$

and

$$
H_{r, d}=\left\{m_{\frac{d(d-1)}{2}+1}, \ldots, m_{\frac{d(d+1)}{2}}\right\}=\left\{a_{d} e_{2} \cdots e_{2 r-2}, a_{d-1} e_{2} \cdots e_{2 r-2}, \ldots, a_{1} e_{2} \cdots e_{2 r-2}\right\} .
$$


Example 3.5. We illustrate some of the above ideas using the graph $G_{3,5}$ (see Figure 1). A Gröbner basis for $I_{G_{3,5}}$ is given by

$$
\begin{gathered}
\left\{a_{5} b_{4}-b_{5} a_{4}, a_{5} b_{3}-b_{5} a_{3}, a_{4} b_{3}-b_{4} a_{3}, a_{5} b_{2}-b_{5} a_{2}, a_{4} b_{2}-b_{4} a_{2},\right. \\
a_{3} b_{2}-b_{3} a_{2}, a_{5} b_{1}-b_{5} a_{1}, a_{4} b_{1}-b_{4} a_{1}, a_{3} b_{1}-b_{3} a_{1}, a_{2} b_{1}-b_{2} a_{1}, \\
\left.a_{5} e_{2} e_{4}-b_{5} e_{1} e_{3}, a_{4} e_{2} e_{4}-b_{4} e_{1} e_{3}, a_{3} e_{2} e_{4}-b_{3} e_{1} e_{3}, a_{2} e_{2} e_{4}-b_{2} e_{1} e_{3}, a_{1} e_{2} e_{4}-b_{1} e_{1} e_{3}\right\} .
\end{gathered}
$$

Using the graded reverse lex order, $\operatorname{in}\left(I_{G_{3,5}}\right)$ is generated by the elements of $\mathcal{G}_{3,5}$ :

$$
\left\{a_{5} b_{4}, a_{5} b_{3}, a_{4} b_{3}, a_{5} b_{2}, a_{4} b_{2}, a_{3} b_{2}, a_{5} b_{1}, a_{4} b_{1}, a_{3} b_{1}, a_{2} b_{1}, a_{5} e_{2} e_{4}, a_{4} e_{2} e_{4}, a_{3} e_{2} e_{4}, a_{2} e_{2} e_{4}, a_{1} e_{2} e_{4}\right\} \text {, }
$$

which we have ordered from smallest to largest.

Theorem 3.6. Fix integers $r \geq 3$ and $d \geq 2$. Then $\operatorname{in}\left(I_{G_{r, d}}\right)$ has linear quotients with respect to $\left\{m_{1}, \ldots, m_{\frac{d(d+1)}{2}}\right\}$. Furthermore

$$
\left(n_{1}, \ldots, n_{\frac{d(d+1)}{2}}\right)=(0, \underbrace{1,1}_{2}, \underbrace{2,2,2}_{3}, \ldots, \underbrace{d-2, d-2, \ldots, d-2}_{d-1}, \underbrace{d-1, d-1, \ldots, d-1}_{d})
$$

where $n_{p}$ is the number of minimal number of generators of $\left\langle m_{1}, \ldots, m_{p-1}\right\rangle:\left\langle m_{p}\right\rangle$.

Proof. Let $I(p)=\left\langle m_{1}, \ldots, m_{p-1}\right\rangle:\left\langle m_{p}\right\rangle$, for $p \in\left\{2, \ldots, \frac{d(d+1)}{2}\right\}$. A generating set of $I(p)$ is given by:

$$
I(p)=\left\langle\frac{L C M\left(m_{1}, m_{p}\right)}{m_{p}}, \frac{L C M\left(m_{2}, m_{p}\right)}{m_{p}}, \ldots, \frac{L C M\left(m_{p-1}, m_{p}\right)}{m_{p}}\right\rangle .
$$

We show that this generating set consists of linear forms. We consider four cases depending on the form of $m_{p}$.

Case 1: If $m_{p}=a_{d} b_{j}$, then

$$
I(p)=\left\langle a_{d} b_{d-1}, \ldots, a_{d} b_{j+1}, \ldots, a_{j} b_{j+1}\right\rangle:\left\langle a_{d} b_{j}\right\rangle=\left\langle b_{d-1}, \ldots, b_{j+1}\right\rangle .
$$

Case 2: If $m_{p}=a_{j+1} b_{j}$ with $j+1<d$, then

$$
I(p)=\left\langle a_{d} b_{d-1}, a_{d} b_{d-2}, a_{d-1} b_{d-2}, \ldots, a_{d} b_{j}, \ldots, a_{j+2} b_{j}\right\rangle:\left\langle a_{j+1} b_{j}\right\rangle=\left\langle a_{j+2}, \ldots, a_{d}\right\rangle .
$$

Case 3: If $m_{p}=a_{i} b_{j}$ with $i-j \geq 2$ and $i<d$, then

$$
I(p)=\left\langle a_{d} b_{d-1}, a_{d} b_{d-2}, \ldots, a_{d} b_{j}, \ldots, a_{i+1} b_{j}\right\rangle:\left\langle a_{i} b_{j}\right\rangle=\left\langle a_{d}, \ldots, a_{i+1}, b_{i-1}, \ldots, b_{j+1}\right\rangle .
$$

Case 4: If $m_{p}=a_{i} e_{2} e_{4} \cdots e_{2 r-2}$ for $1 \leq i \leq d$, then

$$
\begin{aligned}
I(p)= & \left\langle a_{d} b_{d-1}, a_{d} b_{d-2}, a_{d-1} b_{d-2}, \ldots a_{d} b_{1}, a_{d-1} b_{1}, \ldots, a_{2} b_{1},\right. \\
& \left.a_{d} e_{2} e_{4} \cdots e_{n-2}, \ldots, a_{i+1} e_{2} e_{4} \cdots e_{2 r-2}\right\rangle:\left\langle a_{i} e_{2} e_{4} \cdots e_{2 r-2}\right\rangle \\
= & \begin{cases}\left\langle a_{d}, \ldots, a_{2}\right\rangle & \text { for } i=1 \\
\left\langle a_{d}, \ldots, a_{i+1}, b_{i-1}, \ldots, b_{1}\right\rangle & \text { for } 1<i<d \\
\left\langle b_{d-1}, b_{d-2}, \ldots, b_{1}\right\rangle & \text { for } i=d\end{cases}
\end{aligned}
$$

From the four cases, we see that in $\left(I_{G_{r, d}}\right)$ has linear quotients.

Let $n_{p}$ be the number of minimal generators for $I(p)$ as computed above. In Cases 1-3, we have $n_{p}=d-j-1$ where $j$ is the subscript of the $b$-variable, and in Case 4 , we have 
$n_{p}=d-1$. From our order of $m_{1}, \ldots, m_{\frac{d(d-1)}{2}}$, the first $\frac{d(d-1)}{2}$ generators, the subscript $j$ of the $b$-variable follows the order

$$
d-1, \underbrace{d-2, d-2}_{2}, \underbrace{d-3, d-3, d-3}_{3}, \ldots, \underbrace{1,1,1, \ldots, 1}_{d-1} .
$$

The statement now follows.

Theorem 3.7. Fix integers $r \geq 3$ and $d \geq 2$. The graded Betti numbers of in $\left(I_{G_{r, d}}\right)$ are:

$$
\begin{aligned}
& \beta_{i, i+2}\left(\operatorname{in}\left(I_{G_{r, d}}\right)\right)=(i+1)\left(\begin{array}{c}
d \\
i+2
\end{array}\right) \text { for } 0 \leq i \leq d-2, \\
& \beta_{i, i+r}\left(\operatorname{in}\left(I_{G_{r, d}}\right)\right)=d\left(\begin{array}{c}
d-1 \\
i
\end{array}\right) \text { for } 0 \leq i \leq d-1,
\end{aligned}
$$

and $\beta_{i, i+j}\left(\operatorname{in}\left(I_{G_{r, d}}\right)\right)=0$ everywhere else.

Proof. Theorem 3.6 shows in $\left(I_{G_{r, d}}\right)$ has linear quotients with respect to the ordered set $\left\{m_{1}, \ldots, m_{\frac{d(d+1)}{2}}\right\}$. By Theorem 2.4, $\beta_{i, i+j}\left(\operatorname{in}\left(I_{G_{r, d}}\right)\right) \neq 0$ only if $j \in\{2, r\}$.

We now consider the case $j=2$. By Theorem 3.6 the first $\frac{d(d-1)}{2}$ degree two generators satisfy $\left(n_{1}, \ldots, n_{\frac{d(d-1)}{2}}\right)=(0,1,1,2,2,2, \ldots, \underbrace{d-2, d-2, \ldots, d-2}_{d-1})$. By Theorem 2.4 it follows that:

$$
\begin{aligned}
\beta_{i, i+2}\left(\operatorname{in}\left(I_{G_{r, d}}\right)\right) & =\sum_{p=1}^{\frac{d(d-1)}{2}}\left(\begin{array}{c}
n_{p} \\
i
\end{array}\right)=\left(\begin{array}{l}
0 \\
i
\end{array}\right)+2\left(\begin{array}{l}
1 \\
i
\end{array}\right)+3\left(\begin{array}{l}
2 \\
i
\end{array}\right)+\ldots+(d-1)\left(\begin{array}{c}
d-2 \\
i
\end{array}\right) \\
& =\sum_{q=1}^{d-1} q\left(\begin{array}{c}
q-1 \\
i
\end{array}\right)=(i+1) \sum_{q=1}^{d-1}\left(\begin{array}{c}
q \\
i+1
\end{array}\right)=(i+1)\left(\begin{array}{c}
d \\
i+2
\end{array}\right) .
\end{aligned}
$$

The second-to-last equality follows from the identity $k\left(\begin{array}{l}a \\ k\end{array}\right)=a\left(\begin{array}{l}a-1 \\ k-1\end{array}\right)$, and the last equality follows from the identity $\sum_{j=1}^{a}\left(\begin{array}{l}j \\ b\end{array}\right)=\left(\begin{array}{l}a+1 \\ b+1\end{array}\right)$ for $b>0$.

When $j=r$, there are $d$ degree $r$ generators each with $n_{p}=d-1$, so we obtain:

$$
\beta_{i, i+r}\left(\operatorname{in}\left(I_{G_{r, d}}\right)\right)=\sum_{p=\frac{d(d-1)}{2}+1}^{\frac{d(d+1)}{2}}\left(\begin{array}{c}
n_{p} \\
i
\end{array}\right)=d\left(\begin{array}{c}
d-1 \\
i
\end{array}\right) .
$$

This now completes the proof.

We can now give a new proof of [2, Theorem 5.1].

Corollary 3.8. Let $K_{2, d}$ be the complete bipartite graph with $d \geq 2$. Then in $\left(I_{K_{2, d}}\right)$ has linear quotients, and the graded Betti numbers of $I_{K_{2, d}}$ are given by:

$$
\beta_{i, i+2}\left(I_{K_{2, d}}\right)=\beta_{i, i+2}\left(\operatorname{in}\left(I_{K_{2, d}}\right)\right)=(i+1)\left(\begin{array}{c}
d \\
i+2
\end{array}\right) \text { for } 0 \leq i \leq d-2,
$$

and $\beta_{i, i+j}\left(I_{K_{2, d}}\right)=0$ everywhere else. 
Proof. By Remark 3.4, $\left\{a_{i} b_{j}-b_{i} a_{j} \mid 1 \leq i<j \leq d\right\}$ is a Gröbner basis of the toric ideal $I_{K_{2, d}}$ for any monomial order, so that $\operatorname{in}\left(I_{K_{2, d}}\right)=\left\langle F_{d}\right\rangle$. By repeating the arguments of Theorems 3.6 and 3.7, using only the members of $F_{d}$, in $\left(I_{K_{2, d}}\right)$ has linear quotients and graded Betti numbers:

$$
\beta_{i, i+2}\left(\operatorname{in}\left(I_{K_{2, d}}\right)\right)=(i+1)\left(\begin{array}{c}
d \\
i+2
\end{array}\right) \text { for } 0 \leq i \leq d-2,
$$

and $\beta_{i, i+k}\left(\operatorname{in}\left(I_{K_{2, d}}\right)\right)=0$ for all $k \neq 2$. By Theorem 2.5, $\beta_{i, i+k}\left(I_{K_{2, d}}\right)=\beta_{i, i+k}\left(\operatorname{in}\left(I_{K_{2, d}}\right)\right)=0$ for all $k \neq 2$. If we apply Lemma 2.6 , we get $\beta_{i, j}\left(I_{K_{2, d}}\right)=\beta_{i, j}\left(\operatorname{in}\left(I_{K_{2, d}}\right)\right)$ for all $i, j \geq 0$.

We now come to our main theorem.

Theorem 3.9. Fix integers $r \geq 3$ and $d \geq 2$. Then

$$
\beta_{i, i+j}\left(I_{G_{r, d}}\right)=\beta_{i, i+j}\left(\operatorname{in}\left(I_{G_{r, d}}\right)\right) \text { for all } i, j \geq 0 .
$$

In particular, $\beta_{i, i+j}\left(I_{G_{r, d}}\right)$ can be computed using Theorem 3.7.

Proof. By Theorems 2.5 and 3.7, we have $\beta_{i, i+j}\left(I_{G_{r, d}}\right)=\beta_{i, i+j}\left(\operatorname{in}\left(I_{G_{r, d}}\right)\right)=0$ for all $j \neq 2, r$. By Remark 3.4, $I_{K_{2, d}}=\left\langle a_{i} b_{j}-b_{i} a_{j} \mid 1 \leq i<j \leq d\right\rangle$. By Corollary 3.3, the generators of $I_{K_{2, d}}$ are the same as the degree two generators of $I_{G_{r, d}}$. Because both $I_{K_{2, d}}$ and $I_{G_{r, d}}$ have no linear generators, we have $\beta_{i, i+2}\left(I_{G_{r, d}}\right)=\beta_{i, i+2}\left(I_{K_{2, d}}\right)$ for all $i \geq 0$, i.e., they have the same linear strand. By Theorems 3.7 and 3.8, $\beta_{i, i+2}\left(\operatorname{in}\left(I_{G_{r, d}}\right)\right)=\beta_{i, i+2}\left(I_{K_{2, d}}\right)$, which gives $\beta_{i, i+2}\left(I_{G_{r, d}}\right)=\beta_{i, i+2}\left(\operatorname{in}\left(I_{G_{r, d}}\right)\right)$ for all $i \geq 0$. The conclusion now follows by applying Lemma 2.6 since we have shown that $\beta_{i, i+j}\left(I_{G_{r, d}}\right)=\beta_{i, i+j}\left(\operatorname{in}\left(I_{G_{r, d}}\right)\right.$ for all $j \neq r$.

\section{Consequences}

In this section we record some consequences of Theorem 3.9 ,

We first deduce some bounds on the regularity and projective dimension for the toric ideal of any graph $G$ using Theorem 3.9. The (Castelnuovo-Mumford) regularity of any ideal $I$ is defined to be $\operatorname{reg}(I)=\max \left\{j-i \mid \beta_{i, j}(I) \neq 0\right\}$, while the projective dimension is $\operatorname{pdim}(I)=\max \left\{i \mid \beta_{i, j}(I) \neq 0\right\}$. Recall that $H$ is an induced subgraph of $G$ if there exits a subset $W \subseteq V(G)$ such that $H=(W, E(W))$ where $E(W)=\{e \in$ $E(G) \mid e \subseteq W\}$.

Theorem 4.1. Let $G$ be a graph, and suppose that $G$ has an induced subgraph of the form $H=G_{r_{1}, d_{1}} \sqcup G_{r_{2}, d_{2}} \sqcup \cdots \sqcup G_{r_{s}, d_{s}}$, i.e., s disjoint subgraphs of the form $G_{r, d}$ with $r \geq 3$ and $d \geq 2$. Then

(i) $\operatorname{reg}\left(I_{G}\right) \geq r_{1}+\cdots+r_{s}-s+1$, and

(ii) $\operatorname{pdim}\left(I_{G}\right) \geq d_{1}+\cdots+d_{s}-1$.

Proof. By Theorem 3.9, $\operatorname{reg}\left(I_{G_{r, d}}\right)=r$ and $\operatorname{pdim}\left(I_{G_{r, d}}\right)=d-1$. Now apply [1, Theorem 3.7].

Next, we show that $R / I_{G_{r, d}}$ is Cohen-Macaulay.

Theorem 4.2. Fix integers $r \geq 3$ and $d \geq 2$, and let $R=k\left[E\left(G_{r, d}\right)\right]$. Then $R / I_{G_{r, d}}$ is a Cohen-Macaulay ring with Krull dimension $\operatorname{dim}\left(R / I_{G_{r, d}}\right)=d+2 r-2$. 
Proof. By Corollary 3.3 the initial ideal of $I_{G_{r, d}}$ with respect to any monomial order is a square-free monomial ideal. A result of Sturmfels [23, Proposition 13.15] implies that $R / I_{G_{r, d}}$ is normal, so thus by a result of Hochster (see [15, 10] or [9, Theorem 5.17]), $R / I_{G_{r, d}}$ is a Cohen-Macaulay ring.

Since $R / I_{G_{r, d}}$ is Cohen-Macaulay, the Auslander-Buchsbaum formula [5, Section 1.3] implies $\operatorname{dim}\left(R / I_{G_{r, d}}\right)=\operatorname{dim}(R)-\operatorname{pdim}\left(R / I_{G_{r, d}}\right)$. Here the Krull dimension of $R$ equals the number of edges in $G_{r, d}$, i.e., $\operatorname{dim}(R)=2 d+2 r-2$. Since $\operatorname{pdim}\left(R / I_{G_{r, d}}\right)=d$ by Theorem 4.1, the conclusion now holds.

We can also use Theorem 3.9 to compute the Hilbert series of $k\left[E\left(G_{r, d}\right)\right] / I_{G_{r, d}}$.

Theorem 4.3. Fix integers $r \geq 3$ and $d \geq 2$, and let $R=k\left[E\left(G_{r, d}\right)\right]$. Then the Hilbert series of $R / I_{G_{r, d}}$ is

$$
H S_{R / I_{G_{r, d}}}(t)=\frac{1+d t+d t^{2}+\cdots+d t^{r-1}}{(1-t)^{d+2 r-2}} .
$$

In particular, the h-vector of $R / I_{G_{r, d}}$ is $(1, d, \ldots, d)$.

Proof. Recall that $\beta_{i+1, j}\left(R / I_{G_{r, d}}\right)=\beta_{i, j}\left(I_{G_{r, d}}\right)$ for all $i, j \geq 0$. In particular, Theorem 3.9, also gives the graded Betti numbers of the quotient $R / I_{G_{r, d}}$, where $\beta_{0,0}\left(R / I_{G_{r, d}}\right)=1$.

By [9, Proposition 4.27] the Hilbert series satisfies

$$
H S_{R / I_{G_{r, d}}}(t)=\frac{\sum_{i}(-1)^{i}\left(\sum_{j} \beta_{i, j} t^{j}\right)}{(1-t)^{2 d+2 r-2}}
$$

where $\beta_{i, j}=\beta_{i, j}\left(R / I_{G_{r, d}}\right)$. Because $\operatorname{dim}\left(R / I_{G_{r, d}}\right)=d+2 r-2$ by Theorem 4.2, we must divide out $(1-t)^{d}$ from the numerator to write the Hilbert series in lowest terms. Expanding the expression in the numerator yields:

$$
\begin{aligned}
\sum_{i}(-1)^{i}\left(\sum_{j} \beta_{i, j} t^{j}\right) & =1+\sum_{i=1}^{d-1}(-1)^{i} \cdot i \cdot\left(\begin{array}{c}
d \\
i+1
\end{array}\right) t^{i+1}+\sum_{i=1}^{d}(-1)^{i} \cdot d \cdot\left(\begin{array}{c}
d-1 \\
i-1
\end{array}\right) t^{i+r-1} \\
& =1+\sum_{i=1}^{d}(-1)^{i+1}(i-1)\left(\begin{array}{c}
d \\
i
\end{array}\right) t^{i}-d t^{r}(1-t)^{d-1}
\end{aligned}
$$

where we applied an index shift in the first sum. If we now apply the identity $(i-1)\left(\begin{array}{l}d \\ i\end{array}\right)=$ $(d-1)\left(\begin{array}{c}d-1 \\ i-1\end{array}\right)-\left(\begin{array}{c}d-1 \\ i\end{array}\right)$ we get

$$
1+(d-1) \sum_{i=1}^{d}\left((-1)^{i+1}\left(\begin{array}{c}
d-1 \\
i-1
\end{array}\right) t^{i}\right)-\sum_{i=1}^{d}\left((-1)^{i+1}\left(\begin{array}{c}
d-1 \\
i
\end{array}\right) t^{i}\right)-d t^{r}(1-t)^{d-1}
$$




$$
\begin{aligned}
& =1+(d-1)(1-t)^{d-1} t+(1-t)^{d-1}-1-d t^{r}(1-t)^{d-1} \\
& =(1-t)^{d-1}\left[1+(d-1) t-d t^{r}\right] \\
& =(1-t)^{d-1}\left[\left(1-t^{r}\right)+t(d-1)\left(1-t^{r-1}\right)\right] \\
& =(1-t)^{d-1}\left[(1-t)\left(1+\cdots+t^{r-1}\right)+t(d-1)(1-t)\left(1+\cdots+t^{r-2}\right)\right] \\
& =(1-t)^{d}\left[1+d t+d t^{2}+\cdots+d t^{r-1}\right] .
\end{aligned}
$$

The conclusion follows.

Remark 4.4. Stanley [22, Conjecture 4] conjectured that if $S$ is a graded Cohen-Macaulay domain, then the $h$-vector of $S$ was unimodal, i.e., there exists an index $j$ such that $h_{i} \leq h_{i+1}$ for all $0 \leq i<j$ and $h_{i} \geq h_{i+1}$ for $i \geq j$. Since toric ideals are always prime, Theorem 4.2 implies that $K\left[E\left(G_{r, d}\right)\right] / I_{G_{r, d}}$ is a graded Cohen-Macaulay domain, and Theorem 4.3 shows that these domains satisfy Stanley's conjecture. Note that there is some ambiguity in the literature regarding the precise statement and status of Stanley's conjecture; in particular, see [17] and [3], but also refer to the appendix in the corrected version of [3] on the arXiv (arXiv:1505.07377v3 [math.CO]).

\section{REFERENCES}

[1] S. Beyarslan, H.T. Hà, A. O'Keefe, Algebraic properties of toric rings of graphs. Preprint (2017) arXiv: 1703.08270

[2] J. Biermann, A. O'Keefe, A. Van Tuyl, Bounds on the regularity of toric ideals of graphs. Adv. in Appl. Math. 85 (2017), 84-102.

[3] B. Braun, Unimodality problems in Ehrhart theory. Recent trends in combinatorics, 687-711, IMA Vol. Math. Appl., 159, Springer, [Cham], 2016.

[4] B. Braun and R. Davis, Ehrhart series, unimodality, and integrally closed reflexive polytopes, Ann. Comb. 20 (2016), no. 4, 705-717.

[5] W. Bruns, J. Herzog, Cohen-Macaulay rings (Revised Edition). Cambridge University Press, 1998.

[6] A. Conca, M. Varbaro, Square-free Groebner degenerations. Preprint (2018) arXiv:1805.11923

[7] A. Corso, U. Nagel, Monomial and toric ideals associated to Ferrers graphs. Trans. Amer. Math. Soc. 361 (2009), 1371-1395.

[8] A. D'Alì, Toric ideals associated with gap-free graphs. J. Pure Appl. Algebra 219 (2015), 3862-3872.

[9] V. Ene, J. Herzog, Gröbner bases in commutative algebra. Graduate Studies in Mathematics, 130. American Mathematical Society, 2012.

[10] I. Gitler, C.E. Valencia, Multiplicities of edge subrings. Discrete Math. 302 (2005), 107-123.

[11] D. Grayson, M. Stillman, Macaulay2, a software system for research in algebraic geometry. Available at http://www.math.uiuc.edu/Macaulay2/

[12] J. Herzog, Y. Takayama, Resolutions by mapping cones. The Roos Festschrift volume, 2. Homology Homotopy Appl. 4 (2002), part 2, 277-294.

[13] T. Hibi, A. Higashitani, K. Kimura, A. O'Keefe, Depth of edge rings arising from finite graphs. Proc. Amer. Math. Soc. 139 (2011), 3807-3813.

[14] T. Hibi, A. Higashitani, K. Kimura, A. O'Keefe, Depth of initial ideals of normal edge rings. Comm. Algebra 42 (2014), 2908-2922.

[15] M. Hochster, Rings of invariants of tori, Cohen-Macaulay rings generated by monomials, and polytopes. Ann. of Math. 96 (1972), 318-337.

[16] M. Katzman, Bipartite graphs whose edge algebras are complete intersections. J. Algebra 220 (1999), $519-530$.

[17] M. Michałek, Normal and Very Ample Polytopes - old and new open problems. To appear Oberwolfach Reports (2017). DOI : 10.4171/OWR/2017/44 
[18] H. Ohsugi, T. Hibi, Koszul bipartite graphs. Adv. in Appl. Math. 22 (1999), 25-28.

[19] I. Peeva, Graded syzygies. Algebra and Applications, 14. Springer-Verlag London, Ltd., London, 2011.

[20] E. Reyes, C. Tatakis, A. Thoma, Minimal generators of toric ideals of graphs. Adv. in Appl. Math. 48 (2012), 64-78.

[21] L. Sharifan, M. Varbaro, Graded Betti numbers of ideals with linear quotients. Matematiche (Catania) 63 (2008), 257-265.

[22] R. Stanley, Log-concave and unimodal sequences in algebra, combinatorics, and geometry. Graph theory and its applications: East and West (Jinan, 1986), 500-535, Ann. New York Acad. Sci., 576, New York Acad. Sci., New York, 1989.

[23] B. Sturmfels, Gröbner Bases and Convex Polytopes. University Lecture Series, 8. American Mathematical Society, 1996.

[24] R.H. Villarreal, Rees algebras of edge ideals. Comm. Algebra 23 (1995), 3513-3524.

[25] R.H. Villarreal, Monomial algebras. Monographs and Textbooks in Pure and Applied Mathematics, 238. Marcel Dekker, Inc., New York, 2001.

Department of Mathematics, Cleveland State University Cleveland, OH 44115-2215

E-mail address: f.galetto@csuohio.edu

Department of Mathematics and Statistics, McMaster University, Hamilton, ON, L8S $4 \mathrm{~L} 8$

E-mail address: hofschej@mcmaster.ca, keipergt@mcmaster.ca, kohnec@math.mcmaster.ca, vantuyl@math.mcmaster.ca

Department of Mathematics, Escuela Superior de Física y Matemáticas, Instituto Politécnico Nacional, 07300 Mexico City.

E-mail address: muribep1700@alumno.ipn.mx 